\title{
An Analysis on the Artistic Characteristics of Dehua Porcelain Sculptures in Ming and Qing Dynasties
}

\author{
Zibo Lin ${ }^{1, *}$ Chunji Xie ${ }^{1}$ \\ ${ }^{1}$ Xiamen Academy of Arts and Design, Fuzhou University, Xiamen, Fujian, China \\ *Corresponding author. Email: 1036154192@qq.com
}

\begin{abstract}
Dehua kiln in Fujian Province is a producing area of white porcelain with a long history in China, and Dehua porcelain sculpture, as a representative of Dehua kiln, is well-known in China and foreign countries during the Ming and Qing dynasties, and is also an excellent traditional national culture of China. He Chaozong and $\mathrm{Xu}$ Youyi are two most famous porcelain masters of "He School" and "Xu School" in Dehua. And their artistic styles have still influenced the porcelain and sculpture industries in China and foreign countries. This study explores the artistic characteristics of "He School" and "Xu School" respectively, and compares the two works of the same theme by the two masters, analyzes in detail the uniqueness of the two main schools of Dehua porcelain sculpture in the Ming and Qing Dynasties, and reveals the artistic laws of porcelain sculpture. It is hoped to give Dehua porcelain sculptors inspiration to further promote the Chinese culture and help build a system of Chinese cultural self-confidence and cultural consciousness.
\end{abstract}

Keywords: Dehua porcelain sculpture, Artistic characteristics, He Chaozong and Xu Youyi.

\section{INTRODUCTION}

During the Ming and Qing Dynasties, with the development of Dehua kilns, many porcelain sculpture masters emerged in Dehua region, and the most famous beings were He Chaozong and $\mathrm{Xu}$ Youyi. He Chaozong in the late Ming Dynasty pioneered the unique artistic style of Dehua porcelain sculpture, while $\mathrm{Xu}$ Youyi in the period of the Republic of China did not stick to the achievements of predecessors and formed his own unique artistic style, which further promoted the development of Dehua porcelain sculpture.

\section{DEVELOPMENT OF DEHUA PORCELAIN SCULPTURE IN MING AND QING DYNASTIES}

\subsection{Historical Background}

Dehua kiln ushered in the golden age after the prosperity of that in the Dong and Yuan Dynasties. Although because of the long-term "ban policy on maritime trade or intercourse with foreign countries" implemented by the government, and in the eighth year of Chenghua period, the imperial court moved the Bureau for Foreign Shipping originally located in Quanzhou to Fuzhou, the Quanzhou port, which had been prosperous for 300 years, declined, which seriously affected the overseas sales of Dehua porcelain products and slowed down the development. However, the Western porcelain-making technology was not developed during this period, and the demand for Chinese porcelain in the international market was still huge, and there was a new breakthrough in the downturn of porcelain products in Quanzhou. A large number of porcelain products in Quanzhou were exported abroad through Anhai port and Zhangzhou Yue port by means of folk trade. After realizing the disadvantages of "ban policy on maritime trade or intercourse with foreign countries", the imperial court officially cancelled the "ban policy on maritime trade or intercourse with foreign countries" in the first year of Longqing period. At the same time, it opened a "foreign market" in Yue port, Zhangzhou, allowing traders to have free trade. The porcelain industry in Quanzhou also ushered in a happy time. In the Qing Dynasty, the imperial court implemented a strict border relocation policy in order to make Zheng Chenggong completely lose contact with the 
mainland. Fujian, as Zheng Chenggong's hometown, bore the brunt. Therefore, the export-oriented industries in Fujian coastal areas were greatly impacted, the local private maritime trade in Quanzhou was also deeply affected, and the local porcelain industry was gradually weakened under its influence. A large number of craftsmen had to leave the coast to seek new development in the mainland. With its unique advantages, Dehua kiln in the mainland not only absorbed a large amount of funds from the coast, but also attracted many skilled craftsmen. Dehua kiln entered a period of rapid development. In the 23rd year of Kangxi period, the "ban policy on maritime trade or intercourse with foreign countries" was cancelled, the imperial court of Qing Dynasty set up customs in Xiamen. Xiamen port became the largest maritime trade port in Fujian at that time. A large number of foreign businessmen came to Dehua to customize porcelain, and Dehua kiln wa sought after by foreign businessmen and known overseas for its advantages of low price, strong practicability and high quality.

\subsection{Overview of Dehua Porcelain Industry}

Dehua is rich in kaolin. The clay has high silicon content, low iron content and few impurities. In addition, the glaze ash content in the glaze is also very low, so Dehua porcelain works show a kind of bright texture. The clay in Dehua is the best raw material for making high-quality white porcelain works, but the requirements for forming process and firing process are stricter. After a long time of repeated practice and summary, the kiln workers in the Ming Dynasty finally developed the Chinese white porcelain, which was unique in the history of porcelain and famous in China and foreign countries. This kind of white porcelain had excellent quality, delicate texture, simple and elegant color, bright and lustrous glaze, which was like white and fat jade. Also, it was known as "lard white" and "ivory white". When the French saw Dehua porcelain, they were amazed and called it "Chinese white". In "porcelain elegance", a man of the Qing Dynasty once wrote: "the white porcelain plate exported by Dehua is snow-white, cheap and of good style." He gave Dehua porcelain an objective and high evaluation. Dehua porcelain products are famous for porcelain sculptures, especially religious figures. Its unique artistic charm makes Dehua white porcelain enjoy a very high reputation in China and foreign countries. Among them, the works of $\mathrm{He}$ Chaozong, a porcelain sculptor, are the most popular, and his works are known as the boutique of "Oriental Art". In addition to the "he school" art represented by $\mathrm{He}$ Chaozong, the "Xu school" art represented by Xu Youyi also has a unique artistic charm. "He school" art and "Xu school" art are the two main schools of Dehua porcelain sculpture in the Ming and Qing Dynasties. Next, the research analyzes the artistic characteristics of "He school" art and "Xu school" art, and people can feel their unique artistic charm.

\section{ART OF "HE SCHOOL"}

\subsection{Process Characteristics}

"He school" art is an artistic style represented by He Chaozong. He Chaozong is a famous porcelain sculpture master in Dehua, Quanzhou. There is no detailed record of his birth and death year and ancestral home, and later generations have different opinions. Although it is not clear the birth and death year of He Chaozong, it can be seen from his existing works that the type used by $\mathrm{He}$ Chaozong is "lard white" in Dehua porcelain of the Ming Dynasty. Due to the different proportion of raw materials, Dehua porcelain white can be divided into "lard white", "onion root white", "ivory white", "goose down white" and so on. "Lard white" porcelain body is delicate, glaze is bright and moist, and the body and glaze are integrated, white and moist as fat. It is considered as a highquality product by contemporary Dehua porcelain researchers. Most of He Chaozong's works are on Buddha statues and immortal statues, such as Guanyin, Dharma, Sakyamuni, Maitreya Buddha, etc., and the most famous works are on "Dharma crossing the river" and "Guanyin crossing the sea". He Chaozong combined the characteristics of various schools and integrated the techniques of wood carving, stone carving and clay sculpture into the local porcelain sculpture techniques in Dehua. He innovatively developed the eight porcelain sculpture techniques of "kneading, carving, carving, pasting, connecting, pushing and repairing". In addition to technical innovation, He Chaozong would pay special attention to some details in his creation. First, in terms of form, He Chaozong would understand the relevant information of carved characters in advance, and then made an overall conception according to the different characters of different characters during creation. At the same time, he would also pay attention to expressing the characters' characters through shaping details. Finally, the work presented vivid artistic features with both form and spirit. In the texture of clothes, He Chaozong integrated the line 
features in Chinese painting, such as Wu Daizi's round brush and ink, Cao's sturdy brush and ink. While taking into account the changing details of clothing patterns, he used a knife instead of a pen. The lines were rhythmic, artistic, smooth and natural. The artistic characteristics of "He School" mainly reflect that the lines are smooth and elegant, rich in change, elegant in shape, realistic in shape, coordinated in proportion, natural in appearance, skilled in techniques, dignified and generous, and vivid in charm.

\subsection{Aesthetic Characteristics}

He Chaozong's works are mainly on small porcelain statues of religious figures. Before the Ming Dynasty, most religious sculptures in China were large-scale. After He Chaozong, religious sculptures were mostly small-scale. With the eternal artistic charm of his works, He Chaozong led the artistic wave of small religious sculpture. He Chaozong's works have both form and spirit, vivid charm, dexterity and extraordinary vulgarity. For example, his masterpiece "Dharma crossing the river" embodies such artistic characteristics ("Figure 1"). The statue of Dharma portrayed by $\mathrm{He}$ Chaozong has a round head, wide forehead, thick eyebrows, drooping eyes, tightly locked eyebrows, gentle undulation of the bridge of the nose, light lips, a small amount of short whiskers at the mouth and chin, curled sideburns, thick and long ears drooping to the shoulder, serious expression and thoughtful nod. Dharma has a magnificent posture, strong waist and back, and his hands are folded in his sleeves. His clothes are elegant, smooth and dynamic, like the breeze when crossing the river with reeds. He Chaozong paid special attention to the overall artistic beauty in his creation, vividly showing that the Bodhidharma stood barefoot on the reed, his clothes were floating, his face was calm, his form was natural, his body was relaxed, creating the artistic conception. Another example is the sitting statue of Wenchang, fully reflecting the above aesthetic characteristics ("Figure 2"). Wenchang is shaped with a scholar's crown on his head, two foot bands intertwined with each other and then hung on his shoulder. He has a square face, soft eyebrows, big eyes, phoenix eyes, slow nose, naturally closed lips, short whiskers above his lips, and a small amount of whiskers and hair on his chin. The whole face is simple and soft. Wenchang is dressed in a robe. The jade ruyi lies in his left hand, and his right hand naturally hangs down and hides in his sleeve. The upper and lower straps on his abdomen set off a round belly, sitting upright and dangerous. The clothing patterns are very hierarchical, showing the elegance and splendor and vividly reflecting the unique charm of Wenchang.

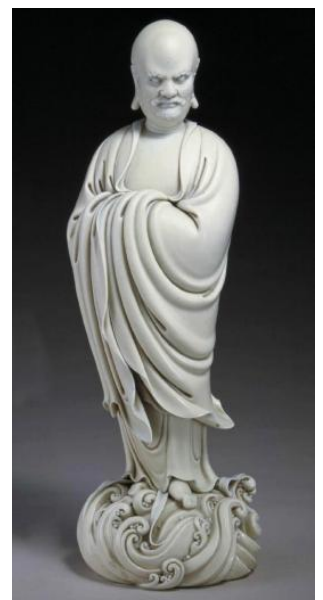

Figure 1 "Dharma crossing the river", He Chaozong.

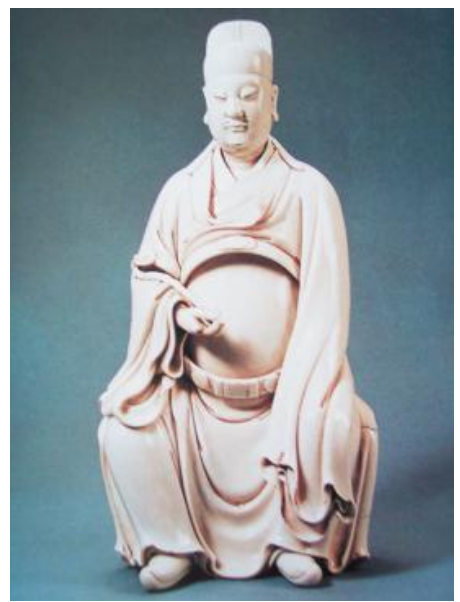

Figure 2 "Sitting statue of Wenchang", He Chaozong.

\section{ART OF "XU SCHOOL"}

\subsection{Process Characteristics}

"Xu school" art is an artistic style represented by $\mathrm{Xu}$ Youyi. Xu Youyi is the 13th descendant of the $\mathrm{Xu}$ family. The $\mathrm{Xu}$ family is a family of the "mountain lake school" of Buddhist sculpture art in Fujian and Guangdong. Xu Liangxi, Xu Youyi's grandfather, is the descendant of the "mountain lake school" sculpture. He is proficient in large-scale temple Buddha sculpture. At the same time, he is also good at micro carving and has superb skills. $\mathrm{Xu}$ Liangxi brought the carving techniques of "mountain lake school" into Dehua area, which had a profound impact on Dehua porcelain sculpture. 
$\mathrm{Xu}$ Youyi, also named Yunlin, dropped out of school at the age of 15 years old. When he came home, he followed his family's clay sculpture and wood carving skills. Later, he followed sculpture master Su Xuejin to learn porcelain sculpture skills. $\mathrm{Su}$ Xuejin is the inheritor of "He School" porcelain sculptures. Influenced by "He School" art, Xu Youyi combined the strengths of various schools and the wood carving skills of local gods and Buddhas, and formed an artistic style with exquisite carving, exquisite modeling, reasonable proportion and gorgeous decoration. In particular, the lines of his works are gentle, smooth and concise. Every carving and drawing is appropriate, and the knife technique is profound and sharp. At the same time, $\mathrm{Xu}$ Youyi innovated and developed techniques such as movable porcelain chain and bead string. With his unique artistic style, Xu Youyi has formed a new trend of Dehua porcelain sculpture art comparable to "He School" art, promoting the development of porcelain sculpture art in Dehua area. The themes of Xu Youyi's works are mostly fairy and Buddha figures, such as Dharma, Buddha, Bodhisattva and so on. In his creation, he used the clay with white color and fine texture, or the clay mixed with fine sand. Most of the glaze used was white and cyan fine flash, and there was also a small amount of milky white glaze. The glaze was thin, moist and transparent, clear and uniform, and the tire glaze was closely combined. Xu Youyi would also pay attention to the characteristic accessories of different character images. The character decoration technology has been known for a time. The carved works are vivid, beautiful and exquisite and of high aesthetic value.

\subsection{Aesthetic Characteristics}

With the development of Dehua porcelain sculpture, porcelain sculpture works are no longer confined to religious beliefs such as immortals and Buddha, and the themes show a diversified development trend. In addition to religious works, $\mathrm{Xu}$ Youyi's works also express national beliefs, and people can see that from $\mathrm{Su} \mathrm{Wu}$, Li Bai, Zhao Yun, Hua Mulan, Wang Zhaojun, etc. Xu Youyi experienced several stages in the production of porcelain sculpture, and his representative porcelain sculpture of "500 arhats" was completed in the stage of most skilled and superb porcelain sculpture techniques, lasting ten years. There are many Buddhist temples in China, but there are few Buddhist temples dedicated to the five hundreds of Arhats. The porcelain sculpture "five hundreds of Arhats" located in Longji temple in Xianyou
County is the only group porcelain sculpture in China. It is said that the porcelain sculptures of Arhats are established according to the list of Arhats in the "stone carving of Arhats in the Qianming courtyard of Jiangyin army" written by Gao Daosu of the Song Dynasty. First, they were designed and drawn by famous traditional Chinese painters, and then made by the brothers of $\mathrm{Xu}$ Youyi, Xu Youguan and Xu Youzan for ten years. These sculptures used the traditional technique of Dehua porcelain sculpture. While paying attention to depicting the distinct personality characteristics of different Arhats, they focused on expressing the thoughts of Arhats, such as being free and unrestrained, helping the world, charity and compassion. The brothers of $\mathrm{Xu}$ Youyi, Xu Youguan and $\mathrm{Xu}$ Youzan made these sculptures by first molding, and then carefully carving them with tools, sculpture, carving and pasting according to their different personality images, so as to make the facial features of arhats vivid and delicate, the clothing patterns and costumes simple and natural, and the postures and expressions different. Then, they glazed the blank body, and finally burned it at high temperature. The whole group sculptures achieved the unity of overall harmony and changeable details, the unity of strong motion and quietness and implicitness, and the unity of internal beauty and formal beauty. It is another milestone in the history of porcelain sculpture. ("Figure 3")

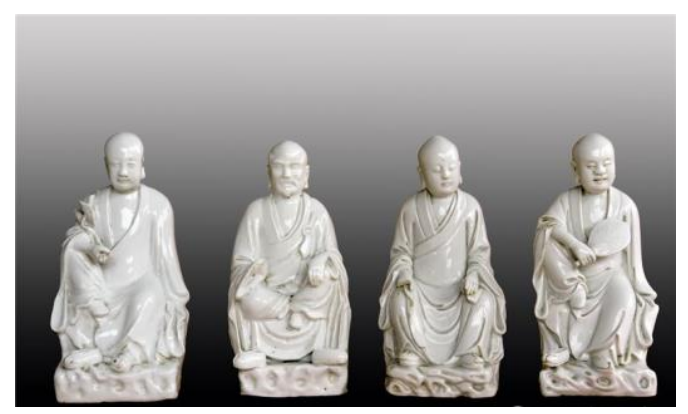

Figure 3 "Eight hundreds of Arhats", Xu Youyi, Xu Youguan and Xu Youzan.

\section{COMPARATIVE STUDY OF ARTISTIC CHARACTERISTICS}

"He school" and "Xu school" are the two most significant artistic styles of porcelain sculpture from the late Ming Dynasty to the Republic of China. Both schools mostly take religious themes as their creative themes, including Guanyin statues. Next, taking He Chaozong's standing statue of Guanyin ("Figure 4") and Xu Youyi's standing statue of Guanyin ("Figure 5") as examples, this 
paper compared and explored the artistic styles of the two schools.

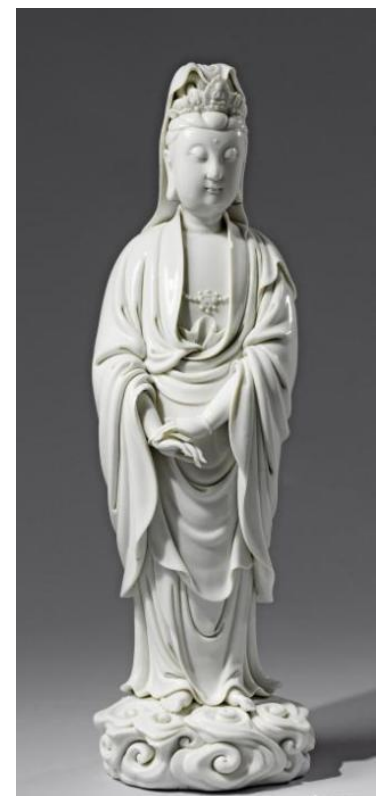

Figure 4 "Standing Statue of Guanyin", He Chaozong.

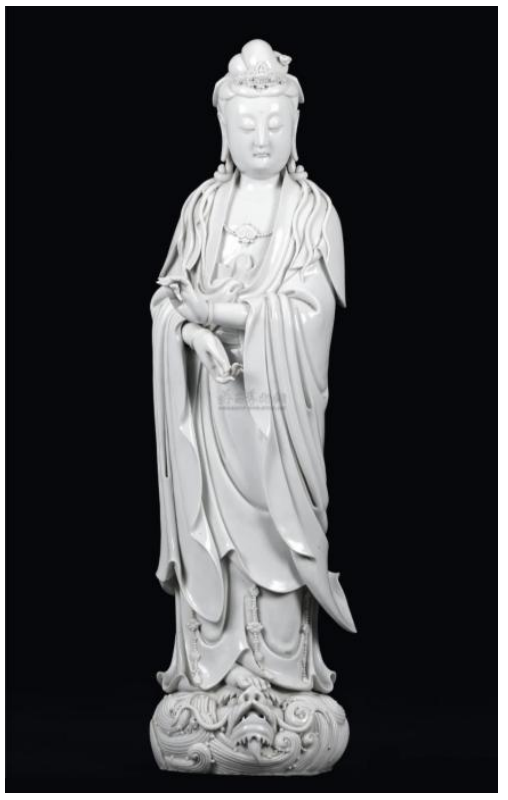

Figure 5 "Statue of Guanyin", Xu Youyi.

\subsection{Facial Features}

Guanyin has the character of great compassion, fraternity and kindness. The facial features of the two standing statues are vivid and delicate. However, there are some differences between the two. He Chaozong's Guanyin statue has a round, more thin and longface, slightly open eyes. He exaggerated to shape the tail of the eyes high, with a large inclination angle. The nose is round, the bridge of the nose fluctuates, the transition is natural, and the lips are full, which shows that Guanyin is full of life, compassion and fraternity. The face of Xu Youyi's Guanyin statue is round and square, with light eyes and tail, gentle inclination angle, more realistic treatment, obvious line feeling of nose undulation, and weak nose performance, showing Guanyin's approachable, kind, gentle, generous and peaceful side. When He Chaozong portrayed the five senses, he tended to express the character through exaggerated artistic techniques, which made the portrayal of the characters vivid and freehand; $\mathrm{Xu}$ Youyi preferred to use the subtle depiction of facial features to match each other, and paid attention to the artistic technique to express the characteristics of the characters, so as to make the characters vivid and delicate.

\subsection{Clothing Lines}

He Chaozong's clothing pleats and textures of Guanyin statues were rich in change, natural and smooth, with a great sense of rhythm. While paying attention to the rich changes of clothing patterns, he also took into account the accuracy of Guanyin's shape and natural movement. The hands of the statue were overlapped in front of the abdomen, showing Guanyin's dignity, meaningful elegance and quietness. The pleats of $\mathrm{Xu}$ Youyi's Guanyin statue were sparse and smooth, the lines were smooth, the clothes were floating, the hands intersected in front of the chest, the left hand was up and the right hand was down, as if they were making Dharma seal, which was natural and flexible. However, clothing patterns made by $\mathrm{Xu}$ Youyi were not as diverse as that of He Chaozong, his sense of rhythm were slightly inferior, the body structure was not as accurate as that of $\mathrm{He}$ Chaozong, the body size was narrower and longer, and the posture was slightly stiff and straight. For example, at the elbow, the folds of He Chaozong's Guanyin were complex, the viewers could still feel the accurate shape of the inside, but Xu Youyi's Guanyin slightly weakened the structure of the elbow.

\subsection{Accessories}

The decoration of He Chaozong's Guanyin mainly had two parts: the exquisite Buddha crown on his hair with plain white yarn and the wishful shaped accessories exposed in front of the chest. In addition, there were few accessories. The body was 
mostly covered by simple clothes. The hair was tied in a bun and hidden in white yarn. Xu Youyi's Guanyin had three main accessories: the exquisite Buddha crown on his hair, Yingluo Ruyi on the chest and Yingluo accessories at the skirt hem. At the same time, the hair naturally hung on the shoulders and fell on the arms. The clothes were more exquisite than those of He Chaozong. In addition to the patchwork clothes, there were shawls. The clothes were layered, and the skirt hem was also equipped with small and fine strings of Yingluo. Xu Youyi's Guanyin decoration was rich and diverse, which greatly increased the delicacy and aesthetic feeling of his works.

Generally speaking, the two works are rare porcelain sculptures, but they have their own emphases. Both show Guanyin's view of fraternity and compassion with skilled and superb techniques.

\section{CONCLUSION}

"He school" art and "Xu school" art are famous in China and foreign countries with their own unique artistic style, and have a great influence on contemporary porcelain sculpture and even sculpture industry. Faced with such excellent cultural achievements of China, the practitioners of Dehua porcelain industry also need to learn to $\mathrm{He}$ Chaozong and $\mathrm{Xu}$ Youyi and hold the attitude of "learning from the ancestors but not from the old rules". They should absorb the essence of traditional porcelain sculpture in Dehua, live in succession, keep pace with the times, make the innovation, and create fine products with the characteristics of the contemporary era.

\section{AUTHORS' CONTRIBUTIONS}

Chunji Xie is the experimental designer and the executive of the experimental research in this study, completing the data analysis and writing the first draft of the paper; Zibo Lin is the project designer and person in charge, guiding the experimental design, data analysis, thesis writing and revision. Both authors read and agreed to the final text.

\section{REFERENCES}

[1] Chen Jianzhong, Chen Lihua, Chen Lifang. History of Dehua Porcelain in China [M]. Shanghai: Shanghai Jiaotong University Press, 2011(7). (in Chinese)
[2] Liu Youzheng. Research on Dehua White Porcelain in China [M]. Beijing. Science Press, 2007. (in Chinese)

[3] Hang Jian. History of Chinese Craft Aesthetics [M]. Beiyue Literature and Art Publishing House, 1994. (in Chinese)

[4] (Ming) Song Yingxing. Exploitation of the works of nature [M]. Annotated by Zhong Guangyan, Guangdong People's Publishing House, 1976. (in Chinese)

[5] Fujian General Chronicles, Volume 93, Art Biography, Ceramics [M]. Collection of the National Library of China. (in Chinese)

[6] (Ming) Chen Maoren. Quannan Magazine [M]. The Commercial Press, 1936. (in Chinese)

[7] Lin Hongfu. China Dehua Ceramic Culture and Works Appreciation. Zhenjiang: Jiangsu University Press, 2014.04. (in Chinese)

[8] Li Min, Wang Zhihui. The Aesthetic Characteristics of Dehua Traditional Porcelain Sculpture Art [J]. Sculpture, 2020, (No. 4). (in Chinese)

[9] Wang Jianhua. Dehua Kiln Guanyin Porcelain Sculpture Collected in the Forbidden City [J]. Cultural Relics, 1994(7). (in Chinese) 